\title{
A panel of rhythm gene polymorphisms is involved in susceptibility to type 2 diabetes mellitus and bipolar disorder
}

\author{
Wenjiao Min ${ }^{1,2}$, Nie Tang ${ }^{3}$, Zhili Zou ${ }^{1}$, Yuexin Chen ${ }^{2}$, Xu Zhang ${ }^{1}$, Yulan Huang ${ }^{1}$, Jinyu Wang ${ }^{1}$, \\ Yaoyin Zhang ${ }^{1}$, Bo Zhou ${ }^{1}$, Xueli Sun ${ }^{2}$ \\ ${ }^{1}$ Psychosomatic Department, Sichuan Provincial People's Hospital, University of Electronic Science and Technology of China, Chinese Academy \\ of Sciences Sichuan Translational Medicine Research Hospital, Chengdu, China; ${ }^{2}$ Mental Health Center, West China University Hospital, Sichuan \\ University, Chengdu, China; ${ }^{3}$ Department of Endocrinology, Sichuan Provincial People's Hospital, Chengdu, China \\ Contributions: (I) Conception and design: W Min, X Sun, B Zhou; (II) Administrative support: B Zhou; (III) Provision of study materials or patients: \\ N Tang, Y Zhang, Z Zou; (IV) Collection and assembly of data: X Zhang, Y Huang, J Wang; (V) Data analysis and interpretation: W Min, Y Chen; (VI) \\ Manuscript writing: All authors; (VII) Final approval of manuscript: All authors. \\ Correspondence to: Bo Zhou, MD. Psychosomatic Department, Sichuan Provincial People's Hospital, University of Electronic Science and Technology \\ of China, Chinese Academy of Sciences Sichuan Translational Medicine Research Hospital, Chengdu, China. Email: jiaojiao1766@163.com; \\ Xueli Sun, MD. Mental Health Center, West China University Hospital, Sichuan University, Chengdu, China. Email: sunxueli58@163.com.
}

Background: Biological rhythm is closely related to health. We aimed to identify the potential correlations of rhythm gene polymorphisms to type 2 diabetes mellitus (DM) or bipolar disorder (BD), which both have many abnormal rhythmic activities, in a sample of Chinese Han origin.

Methods: A total of 136 patients with BD, 166 patients with DM, and 130 healthy controls were collected. We screened 28 single nucleotide polymorphisms (SNPs) located in rhythm genes CLOCK, ARNTL, PER2, PER3, CRY1, and CRY2 respectively. Snapshot typing technology was used for genotyping.

Results: Both the rs10832022-G and rs11022765-A allele frequencies of the ARNTL gene were significantly higher in patients with $\mathrm{DM}$ than in those with $\mathrm{BD}$ (corrected $\mathrm{P}=0.03,0.004$, respectively). The frequency of rs10832022-G, rs1022765-A, and rs11022762-T haplotypes, which was significantly lower in patients with $\mathrm{BD}$ than in controls $(\mathrm{P}=0.003, \mathrm{OR}=0.579)$, was significantly higher in patients with $\mathrm{DM}$ than in those with $\mathrm{BD}(\mathrm{P}=0.0002, \mathrm{OR}=1.878)$. The rs2292910-CC genotypic frequency of the CRY2 gene was significantly higher in patients with $\mathrm{BD}$ than in controls ( $\mathrm{OR}$ of regression $=2.203, \mathrm{P}=0.01$ ), while the frequency of the AA genotype was significantly lower than in patients with $\mathrm{DM}(\mathrm{P}=0.01)$. The frequency of rs1972874-G and rs36124720-G haplotype of the PER2 gene was significantly higher in patients with DM than in controls $(\mathrm{P}=0.01, \mathrm{OR}=1.577)$.

Conclusions: Our study preliminarily suggested that both BD and type 2 DM could be considered as dysrhythmias with different rhythmic genetic backgrounds, which contribute to the early prediction of an individual's susceptibility to different rhythm disorders and early intervention.

Keywords: Type 2 diabetes mellitus (type 2DM); bipolar disorder; rhythm; gene

Submitted Aug 19, 2021. Accepted for publication Oct 19, 2021.

doi: $10.21037 / \mathrm{atm}-21-4803$

View this article at: https://dx.doi.org/10.21037/atm-21-4803

\section{Introduction}

Biological rhythm regulates physiological activities of the body and is closely related to health (1). The suprachiasmatic nucleus (SCN) in the front of the hypothalamus is currently recognized as the rhythm center of mammals (2). The central oscillator of the SCN contains several rhythm genes, including CLOCK, ARNTL, PER1/2/3 and CRY1/2, constituting a "transcriptiontranslation-feedback" loop, which plays an important role in the regulation of circadian activities of the body (3). When 
the rhythm regulation loop turns abnormal, many disorders of rhythm activities may occur, including fluctuations of blood pressure and blood glucose (4).

Type 2 diabetes mellitus (DM) is considered as a typical psychosomatic disease characterized by abnormal blood glucose. Many researchers have found that patients with DM often have circadian rhythm disorder, or hormones of hypothalamic-pituitary-adrenal (HPA) and hypothalamicpituitary-thyroid (HPT) axes secretion rhythm disorder $(5,6)$. Variations in the Rev-erb alpha and Rev-erb beta genes which are both associated with circadian rhythm could affect metabolic changes in DM (7). The rs4580704 single nucleotide polymorphism (SNP) in rhythm gene CLOCK was closely related to the occurrence of type 2 DM. The susceptibility of CC genotype carriers to DM was significantly higher than the other genotype carriers (8).

Bipolar disorder (BD) was a common severe mental disorder characterized by recurrent episodes of hyperthymia and hypothymia or mixed. Epidemiology showed that BD has been the second mental disorder in China with high rates of recurrence, disability and suicide (9). Recent years, $\mathrm{BD}$ has been considered to be a typical emotional rhythm disorder (10). The amplitude of body temperature rhythm decreased in BD patients (11). The rs2304672SNP in PER2 rhythm gene was associated with the susceptibility of BD (12). Besides, similarly to DM, disorder of neuroendocrine axes secretion rhythms (13), and circadian dysrhythmia (14) were also common in patients with BD. Mutations in the CLOCK rhythm gene in mice might lead to hyperglycemia, hyperlipidemia (15), or manic and excitatory behaviors (16). These suggest that the biological rhythm changes are closely related to both $\mathrm{DM}$ and $\mathrm{BD}$. The variation of rhythm genes may affect the susceptibility of individuals to different diseases.

Through previous study on the identification and optimization of atypical symptoms of $\mathrm{BD}$, our research team has proposed the theoretical hypothesis of "stressdysrhythmia", supposing that stress and genetic factors are the pathogenesis of chronic non-infectious diseases, where dysfunctions of rhythms in the body may play an important role in the occurrence and development of disease (17). Different genetic backgrounds of rhythm may be the basis of different rhythm disorders. DM and BD are both common chronic non-infectious diseases, with heavy disease burdens and high rates of disability. They have a high rate of co-morbidity clinically (18), which may affect the treatment and prognosis of patients. Therefore, exploring the influence of rhythm genetic backgrounds on the susceptibilities of $\mathrm{BD}$ and $\mathrm{DM}$ would be helpful for the early prediction and intervention of disease. However, there are still few studies about the association of rhythm gene polymorphisms and BD or DM, with inconsistent conclusions and lack of repeated validation. Moreover, there is a lack of comparison of the differences of rhythm genetic background among different diseases.

Therefore, this study chose the four core circadian rhythm genes CLOCK, ARNTL, PER and CRY, taking healthy people as a control group, to explore the distribution characteristics of rhythm gene SNPs in patients with $\mathrm{DM}$ or $\mathrm{BD}$, to preliminarily verify the influence of different rhythm genetic background on the susceptibilities of different rhythm disorders. We present the following article in accordance with the STREGA reporting checklist (available at https://dx.doi.org/10.21037/atm-21-4803).

\section{Methods}

\section{Subjects}

We enrolled 136 patients with BD and 166 patients with DM from southwest China between December 2018 and December 2019. Patients with BD fulfilled the Diagnostic and Statistical Manual of Mental Disorder, Fourth Edition (DSM-IV) criteria, and the diagnosis was independently assigned by two senior psychiatrists using the Structured Clinical Interview for DSM-IV Disorders-Clinician Version (the Kappa value of the consistency check was greater than 0.8). Patients with DM fulfilled the World Health Organization (WHO) diagnostic criteria for type $2 \mathrm{DM}$ [1999]. The exclusion criteria were as follows: BD group: (I) with other Axis I psychiatric disorders including depression, schizophrenia, anxiety disorder, and substance abuse; (II) with Axis II disorders including personality disorders; (III) with any history of major medical/neurological disorders; (IV) using any drugs that may affect metabolism, such as glucocorticoids in the past 3 months; (V) pregnancy or lactation. For the DM group, the exclusion criteria were: (I) type $1 \mathrm{DM}$ or some other special type of DM; (II) with acute complications of type $2 \mathrm{DM}$ or severe chronic complications; (III) using $\beta$ blockers or glucocorticoids in the past 3 months; (IV) with any history of other endocrine or autoimmune diseases, such as adrenal, thyroid, or gonad disorders; (V) with any history of major medical/neurological disorders; (VI) with any history of schizophrenia and its spectrum disorders, organic mental disorders, or substance abuse; (VII) pregnancy or lactation. 
We also screened 130 race-matched healthy people as controls and ruled out current or past serious physical illnesses or DSM-IV Axis I Disorders through the Structural Clinical Interview for DSM-IV Axis I Disorders, non-patient edition (SCID-NP) (19).

All subjects were unrelated (no blood relationship) and of Chinese Han origin, sharing similar geographic and sociodemographic data. All procedures performed in this study involving human participants were in accordance with the Declaration of Helsinki (as revised in 2013). The study was approved by Institutional Ethics Committee of the Sichuan Provincial People's Hospital [Ethic review (Research) No. 18.2018]. All methods were performed in accordance with the relevant guidelines and regulations, and written informed consent was obtained from each participant.

\section{DNA extraction and genotyping}

Five milliliters of peripheral venous blood were collected from each participant with EDTA anticoagulation on enrollment. Genomic DNAs were extracted from the blood samples using the standard phenol-chloroform method.

We screened 28 single nucleotide polymorphisms (SNPs), which located in rhythm genes CLOCK, ARNTL, PER2, PER3, CRY1, and CRY2, respectively, with the minor allele frequency (MAF) greater than 0.2 for genotyping (Reference website: https://www.stat.ubc.ca/rollin/stats/ssize/caco. html) (Table S1).

Snapshot typing technology was applied to type the 28 SNP sites, and primer sequences were made using online Primer3 software (http://bioinfo.ut.ee/primer3-0.4.0/) (Table S2). The typing process included polymerase chain reaction (PCR), restriction digestion, and multiple single base extension reaction with Snapshot. The extension products were sequenced using ABI3730XL sequencer, and the original data collected were analyzed by Gene Mapper 4.1 (Applied Bio-systems Co., Ltd., USA).

\section{Statistical analysis}

The study used $\chi^{2}$ test to calculate Hardy-Weinberg equilibrium (HWE) and SHEsis software for Linkage disequilibrium (LD) analysis (20). PLINK software was used to conduct logistic regression statistics of SNP alleles, genotypes, and interactions among each group, and $\chi^{2}$ test or ANOVA was used for comparison of the basic demographic data of the three groups in SPSS 18.0 software package. All the tests were two tailed, with alpha set at 0.05 .

\section{Results}

Although there was no significant difference in sex distribution between the DM group and control group $(\mathrm{P}>0.05)$, women greatly outnumbered men in the BD group compared to the $\mathrm{DM}$ and control groups $(\mathrm{P}<0.05)$. The mean age of patients in the $\mathrm{BD}$ group was the youngest $(29.97 \pm 13.460$ years), followed by the control group (38.96 \pm 8.204 years), and that in the DM group was the oldest $(58.81 \pm 10.369$ years).

HWE analysis showed that except rs55794336 $(\mathrm{P}=0.007713)$, the $\mathrm{P}$ value of the other $27 \mathrm{SNP}$ sites were all greater than 0.05 , suggesting that all were consistent with HWE (Table S3). The MAF values of the 27 SNPs in both control and case groups were all similar to those in the gene bank, as were the LD distributions, indicating the samples tested had good reliability and representability.

\section{Comparisons of allele frequencies of SNP sites among three groups}

Both the allele frequencies of rs10832022 and rs11022765 were significantly different between the $\mathrm{BD}$ group and $\mathrm{DM}$ group. The G allele frequency of rs10832022 in the BD group $(36.76 \%)$ was significantly lower than in the DM group (50.00\%), while the A allele frequency $(63.24 \%)$ was significantly higher than in the DM group $(50.00 \%)$ (adjusted $\mathrm{P}=0.03$ ). The A allele frequency of rs11022765 in the BD group (30.15\%) was significantly lower than in the DM group (45.18\%), while the C allele frequency was significantly higher than in the DM group $(69.85 \%$ in the $\mathrm{BD}$ group and $54.82 \%$ in the $\mathrm{DM}$ group, adjusted $\mathrm{P}=0.004$ ).

\section{Comparisons of SNP genotypic distributions between BD group and control group}

Genotypic distributions of the 27 SNPs in patients with $\mathrm{BD}$ and the controls are summarized in Table 1. Both the genotypic distributions of rs10832022 and rs11022765 were statistically different between the BD group and control group $\left(\chi^{2}=8.793, \mathrm{P}=0.01\right.$ for the former, $\chi^{2}=9.009, \mathrm{P}=0.01$ for the latter). Moreover, after the regression analysis including each SNP genotypic distributions, the differences were still statistically significant $(\mathrm{OR}=0.614, \mathrm{P}=0.01$ for rs10832022, $\mathrm{OR}=0.589, \mathrm{P}=0.00$ for $\mathrm{rs} 11022765$ ). However, after the clinical phenotypic regression analysis including 
Table 1 Genotypic distributions of 27 single nucleotide polymorphisms (SNPs) in the bipolar disorder (BD) group and the control group

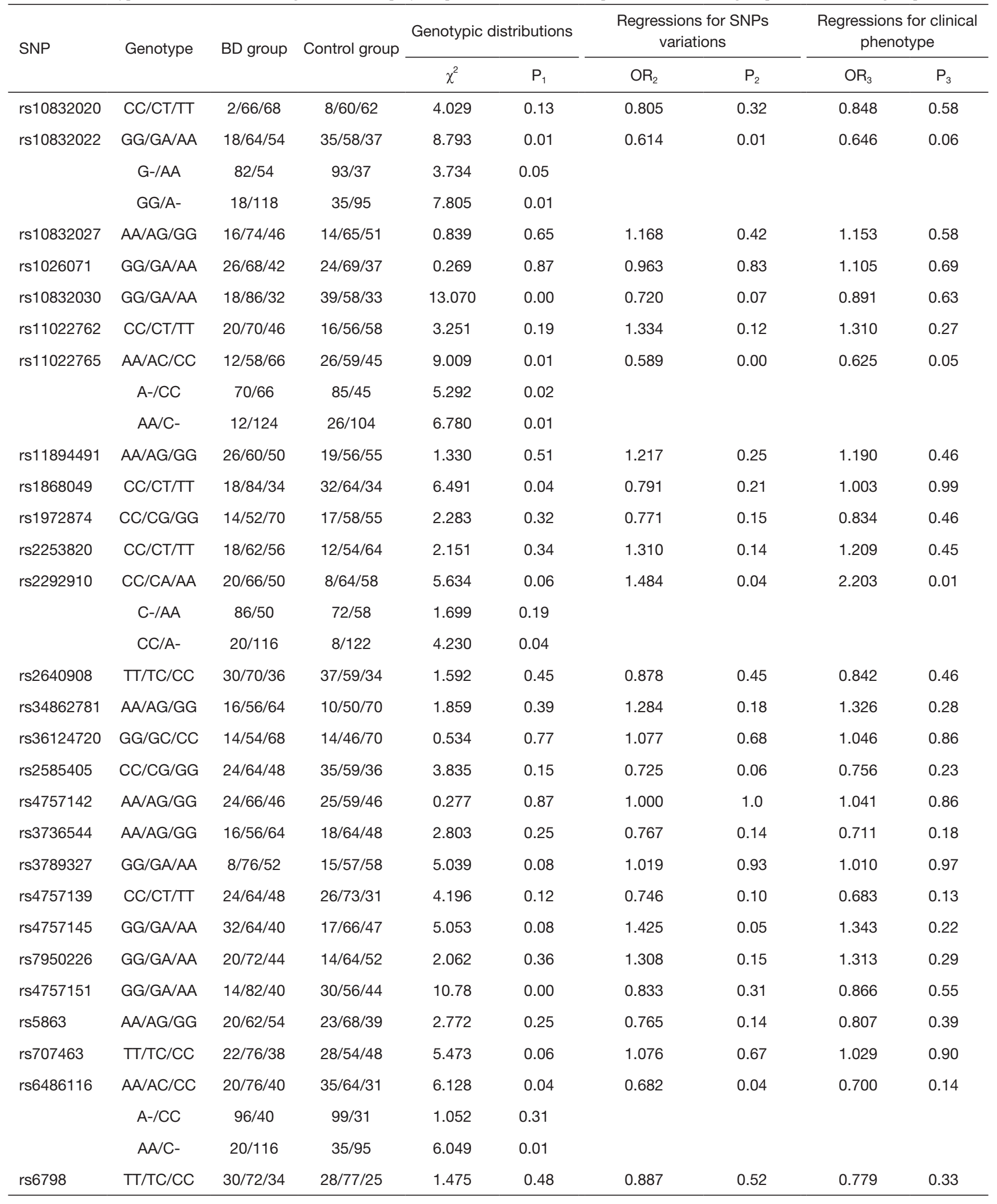


gender and age, the differences were both not statistically significant. The frequencies of the rs10832022 A- genotype and rs11022765 C- genotypes were both much higher in the BD group than in the control group $(86.8 \%$ in the BD group and $73.1 \%$ in the control group, $\chi^{2}=7.805, \mathrm{P}=0.01$ for the former; $91.2 \%$ in the BD group and $80.0 \%$ in the control group, $\chi^{2}=6.78, \mathrm{P}=0.01$ for the latter).

Distributions of the rs2292910 A allele carriers (A-) and CC genotype carriers were both statistically significant between the BD group and the control group $\left(\chi^{2}=4.230\right.$, $\mathrm{P}=0.04)$, and the frequency of the CC genotype in the BD group $(14.7 \%)$ was significantly higher than in the control group (6.7\%). Moreover, after both the regression including each SNP genotypic distributions and clinical phenotypic regression including gender and age, these differences were still statistically significant $(\mathrm{OR}=1.484, \mathrm{P}=0.04$ for the former, $\mathrm{OR}=2.203, \mathrm{P}=0.01$ for the latter).

Rs10832022, rs11022765, and rs11022762 had LD through LD analysis (Figure 1A). Haplotype analysis showed that the distribution frequency of rs10832022-G, rs11022765-A, and rs11022762-T haplotype in the BD group $(30.1 \%)$ was significantly lower than that in the control group $(42.7 \%)\left(\chi^{2}=9.050, P=0.003, O R=0.579,95 \%\right.$ CI: $0.405-0.828)$.

\section{Comparisons of SNP genotypic distributions between DM group and control group}

Genotypic distributions of the 27 SNPs in patients with DM and the controls are summarized in Table 2. The genotypic distribution of rs 1972874 was statistically different between the DM group and control group $\left(\chi^{2}=6.212\right.$, $\mathrm{P}=0.04)$. Moreover, after both the regression including each SNP genotypic distributions and clinical phenotypic regression including gender and age, the difference was still statistically significant $(\mathrm{OR}=0.655, \mathrm{P}=0.02$ for the former, $\mathrm{OR}=0.407, \mathrm{P}=0.02$ for the latter). The frequency of the $\mathrm{CC}$ genotype was much higher in the DM group $(56.5 \%)$ than in the control group $(42.3 \%)\left(\chi^{2}=5.979, \mathrm{P}=0.01\right)$, and the genotypic distribution of rs36124720 was also statistically different between the DM group and the control group $\left(\chi^{2}=6.227, \mathrm{P}=0.04\right)$. While after the regression including each SNP genotypic distributions, the difference was still statistically significant $(\mathrm{OR}=1.512, \mathrm{P}=0.02)$, after the clinical phenotypic regression including gender and age, the difference was not statistically significant. The frequency of the G- genotype was much higher in the DM group (60.2\%) than in the control group $(46.2 \%)\left(\chi^{2}=5.825, \mathrm{P}=0.02\right)$.
The distributions of rs7950226 G allele carriers (G-) and AA genotype carriers were statistically significant between the DM group and control group $\left(\chi^{2}=4.004, P=0.04\right)$, and the frequency of the G- genotype in the DM group $(71.1 \%)$ was significantly higher than in the control group (60.0\%). Moreover, after both regression including each SNP genotypic distributions and clinical phenotypic regression including gender and age, the difference was still statistically significant $(\mathrm{OR}=1.456, \mathrm{P}=0.04$ for the former, $\mathrm{OR}=2.909$, $\mathrm{P}=0.01$ for the latter).

Rs1972874 and rs36124720 had LD through LD analysis (Figure 1B). Haplotype analysis showed that the distribution frequency of rs1972874-C and rs36124720-C haplotype in the DM group $(25.9 \%)$ was significantly lower than in the control group $(35.4 \%)\left(\chi^{2}=6.233, \mathrm{P}=0.01, \mathrm{OR}=0.638\right.$, 95\% CI: 0.448-0.909), while the distribution frequency of rs1972874-G and rs36124720-G haplotype in the DM group (38.6\%) was significantly higher than in the control group $(28.5 \%) \quad\left(\chi^{2}=6.608, \mathrm{P}=0.01, \mathrm{OR}=1.577,95 \% \mathrm{CI}\right.$ : $2.223-2.235)$.

\section{Comparisons of SNP genotypic distributions between DM and BD groups}

Genotypic distributions of the 27 SNPs in DM and BD groups are summarized in Table 3. Besides the significant differences of allele frequencies of rs10832022 and rs11022765, the genotypic distributions of the above two SNPs were also both statistically different between the DM group and BD group $\left(\chi^{2}=11.450, \mathrm{P}=0.00\right.$ for the former, $\chi^{2}=15.380, P=0.00$ for the latter). Moreover, after the regression including each SNP genotypic distributions, the differences were still statistically significant $(\mathrm{OR}=1.774$, $\mathrm{P}=0.00$ for rs10832022, $\mathrm{OR}=1.991, \mathrm{P}=0.00$ for $\mathrm{rs} 11022765$ ). However, after the clinical phenotypic regression including gender and age, the differences were not statistically significant.

The frequency of the rs10832022 G- genotype in the DM group (77.1\%) was significantly higher than that in the $\mathrm{BD}$ group $(60.3 \%)\left(\chi^{2}=9.977, \mathrm{P}=0.00\right)$, and the frequency of the GG genotype in the DM group $(22.9 \%)$ was also significantly higher than that in the BD group (13.2\%) $\left(\chi^{2}=4.615, \mathrm{P}=0.03\right)$. The frequency of the rs11022765 Agenotype in the DM group (72.3\%) was significantly higher than in the BD group $(51.5 \%)\left(\chi^{2}=13.890, \mathrm{P}=0.0002\right)$, and the frequency of the AA genotype in the DM group (18.1\%) was also significantly higher than that in the $\mathrm{BD}$ group $(8.8 \%)\left(\chi^{2}=5.341, \mathrm{P}=0.02\right)$. 

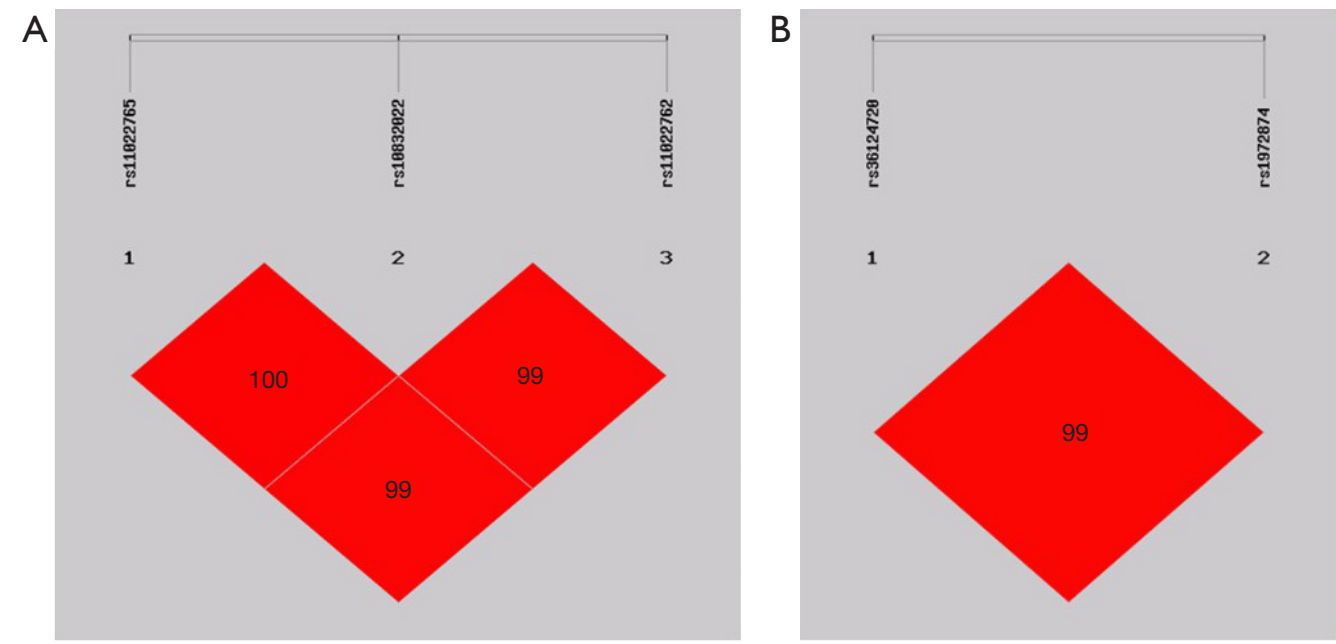

\begin{tabular}{|c|c|c|}
\hline$D^{\prime}$ & $r s 10832022$ & $r s 11022762$ \\
\hline$r s 11022765$ & 1.000 & 1.000 \\
\hline$r s 10832022$ & & 1.000 \\
\hline$r^{2}$ & $r s 10832022$ & $r s 11022762$ \\
\hline$r s 11022765$ & 0.768 & 0.381 \\
\hline$r s 10832022$ & & 0.496 \\
\hline
\end{tabular}

\begin{tabular}{|c|c|}
\hline \multicolumn{2}{|c|}{$r s 1972874-r s 36124720$} \\
\hline$D^{\prime}=1.000$ & $r^{2}=0.223$ \\
\hline
\end{tabular}
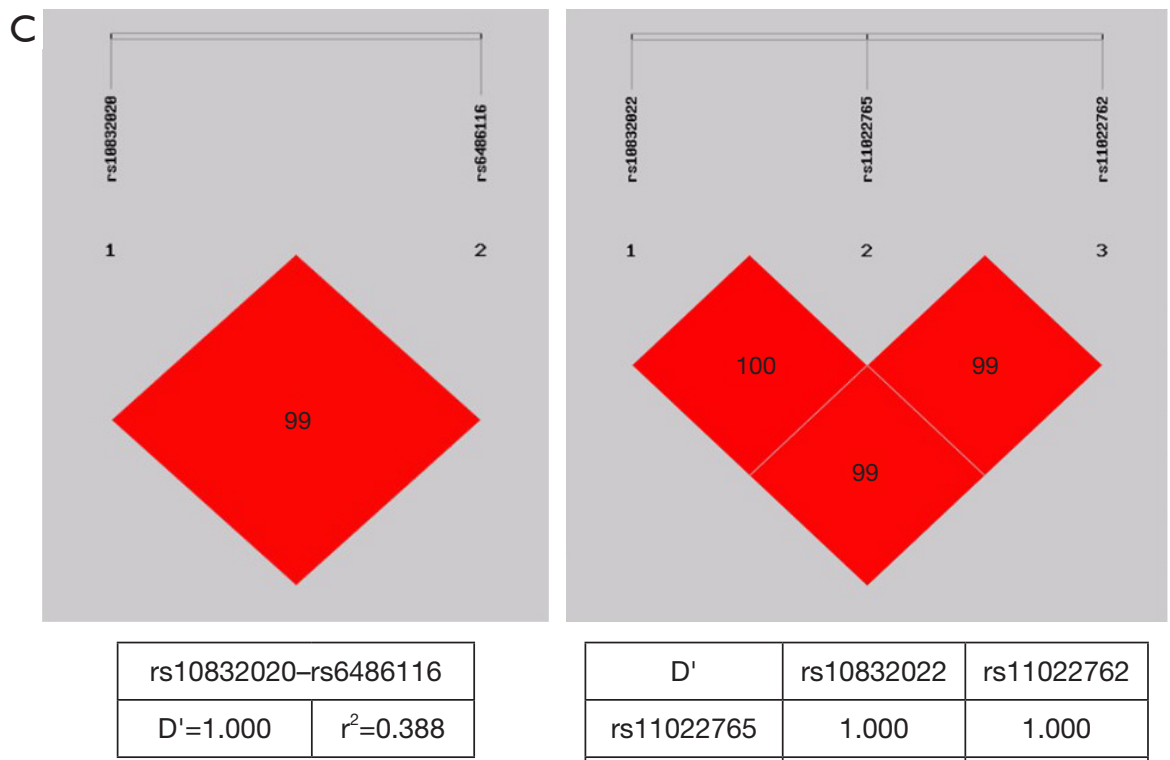

\begin{tabular}{|c|c|c|}
\hline$D^{\prime}$ & $r s 10832022$ & $r s 11022762$ \\
\hline$r s 11022765$ & 1.000 & 1.000 \\
\hline$r s 10832022$ & - & 1.000 \\
\hline$r^{2}$ & $r s 10832022$ & $r s 11022762$ \\
\hline$r s 11022765$ & 0.768 & 0.381 \\
\hline$r s 10832022$ & & 0.496 \\
\hline
\end{tabular}

Figure 1 Linkage disequilibrium (LD) analysis of single nucleotide polymorphisms (SNPs) among groups. (A) LD analysis of SNPs in the bipolar disorder (BD) group and the control group; (B) LD analysis of SNPs in the diabetes mellitus (DM) group and the control group; (C) LD analysis of SNPs in the DM group and the BD group. 
Table 2 Genotypic distributions of 27 single nucleotide polymorphisms (SNPs) in the diabetes mellitus (DM) group and the control group

\begin{tabular}{|c|c|c|c|c|c|c|c|c|c|}
\hline \multirow[t]{2}{*}{ SNP } & \multirow[t]{2}{*}{ Genotype } & \multirow[t]{2}{*}{ DM group } & \multirow[t]{2}{*}{ Control group } & \multicolumn{2}{|c|}{ Genotypic distributions } & \multicolumn{2}{|c|}{$\begin{array}{c}\text { Regressions for SNPs } \\
\text { variations }\end{array}$} & \multicolumn{2}{|c|}{$\begin{array}{l}\text { Regressions for } \\
\text { clinical phenotype }\end{array}$} \\
\hline & & & & $\chi^{2}$ & $\mathrm{P}_{1}$ & $\mathrm{OR}_{2}$ & $\mathrm{P}_{2}$ & $\mathrm{OR}_{3}$ & $\mathrm{P}_{3}$ \\
\hline rs10832022 & GG/GA/AA & $38 / 90 / 38$ & $35 / 58 / 37$ & 2.717 & 0.26 & 1.031 & 0.85 & 1.069 & 0.85 \\
\hline rs10832030 & $A A / A G / G G$ & $28 / 94 / 44$ & 33/58/39 & 4.932 & 0.08 & 0.901 & 0.54 & 1.392 & 0.34 \\
\hline rs11022765 & $\mathrm{AA} / \mathrm{AC} / \mathrm{CC}$ & $30 / 90 / 46$ & $26 / 59 / 45$ & 2.404 & 0.30 & 1.109 & 0.54 & 1.168 & 0.67 \\
\hline rs1868049 & $\mathrm{TT} / \mathrm{TC} / \mathrm{CC}$ & $40 / 82 / 44$ & $34 / 64 / 32$ & 0.225 & 0.89 & 0.925 & 0.64 & 0.977 & 0.94 \\
\hline rs11894491 & $\mathrm{AA} / \mathrm{AG} / \mathrm{GG}$ & $24 / 72 / 70$ & $19 / 56 / 55$ & 0.003 & 1.00 & 1.000 & 1.00 & 1.248 & 0.51 \\
\hline rs2253820 & $\mathrm{CC} / \mathrm{CT} / \mathrm{TT}$ & $10 / 70 / 86$ & $12 / 54 / 64$ & 1.111 & 0.57 & 0.864 & 0.43 & 0.900 & 0.77 \\
\hline rs2292910 & CC/CA/AA & $24 / 54 / 88$ & $8 / 64 / 58$ & 10.790 & 0.01 & 0.998 & 0.99 & 0.757 & 0.44 \\
\hline rs2585405 & $\mathrm{GG} / \mathrm{GC} / \mathrm{CC}$ & $36 / 86 / 44$ & $36 / 59 / 35$ & 1.700 & 0.43 & 0.896 & 0.50 & 0.880 & 0.69 \\
\hline rs2640908 & $\mathrm{TT} / \mathrm{TC} / \mathrm{CC}$ & $32 / 88 / 46$ & $37 / 59 / 34$ & 3.558 & 0.17 & 0.807 & 0.20 & 0.893 & 0.73 \\
\hline rs34862781 & AA/AG/GG & $16 / 78 / 72$ & $10 / 50 / 70$ & 3.207 & 0.20 & 1.356 & 0.10 & 2.752 & 0.01 \\
\hline rs36124720 & $\mathrm{GG} / \mathrm{GC} / \mathrm{CC}$ & $28 / 72 / 66$ & $14 / 46 / 70$ & 6.227 & 0.04 & 1.512 & 0.02 & 1.924 & 0.07 \\
\hline rs4757142 & AA/AG/GG & $22 / 94 / 50$ & $25 / 59 / 46$ & 4.046 & 0.13 & 0.985 & 0.93 & 1.428 & 0.31 \\
\hline rs4757151 & GG/GA/AA & $26 / 80 / 60$ & $30 / 56 / 44$ & 2.643 & 0.27 & 0.828 & 0.25 & 0.692 & 0.26 \\
\hline rs5863 & $\mathrm{AA} / \mathrm{AG} / \mathrm{GG}$ & $26 / 68 / 72$ & 23/68/39 & 5.700 & 0.06 & 0.733 & 0.06 & 0.541 & 0.08 \\
\hline rs6486116 & CC/CA/AA & $34 / 96 / 36$ & $31 / 64 / 35$ & 2.207 & 0.33 & 1.042 & 0.81 & 1.174 & 0.67 \\
\hline rs6798 & $\mathrm{CC} / \mathrm{CT} / \mathrm{TT}$ & $36 / 80 / 50$ & $25 / 77 / 28$ & 3.926 & 0.14 & 0.877 & 0.44 & 0.833 & 0.62 \\
\hline rs707463 & $\mathrm{TT} / \mathrm{TC} / \mathrm{CC}$ & $20 / 92 / 54$ & $28 / 54 / 48$ & 7.306 & 0.03 & 0.898 & 0.53 & 0.968 & 0.92 \\
\hline \multirow[t]{3}{*}{ rs7950226 } & GG/GA/AA & $26 / 92 / 48$ & $14 / 64 / 52$ & 4.473 & 0.11 & 1.456 & 0.04 & 2.909 & 0.01 \\
\hline & G-/AA & $118 / 48$ & $78 / 52$ & 4.004 & 0.04 & & & & \\
\hline & GG/A- & $26 / 140$ & $14 / 116$ & 1.494 & 0.22 & & & & \\
\hline rs3736544 & $\mathrm{AA} / \mathrm{AG} / \mathrm{GG}$ & $22 / 62 / 82$ & $18 / 64 / 48$ & 5.02 & 0.08 & 0.762 & 0.11 & 0.503 & 0.06 \\
\hline rs4757145 & GG/GA/AA & $20 / 76 / 70$ & $17 / 66 / 47$ & 1.107 & 0.58 & 0.854 & 0.37 & 1.078 & 0.84 \\
\hline
\end{tabular}


Table 3 Genotypic distributions of 27 single nucleotide polymorphisms (SNPs) in the diabetes mellitus (DM) group and the bipolar disorder (BD) group

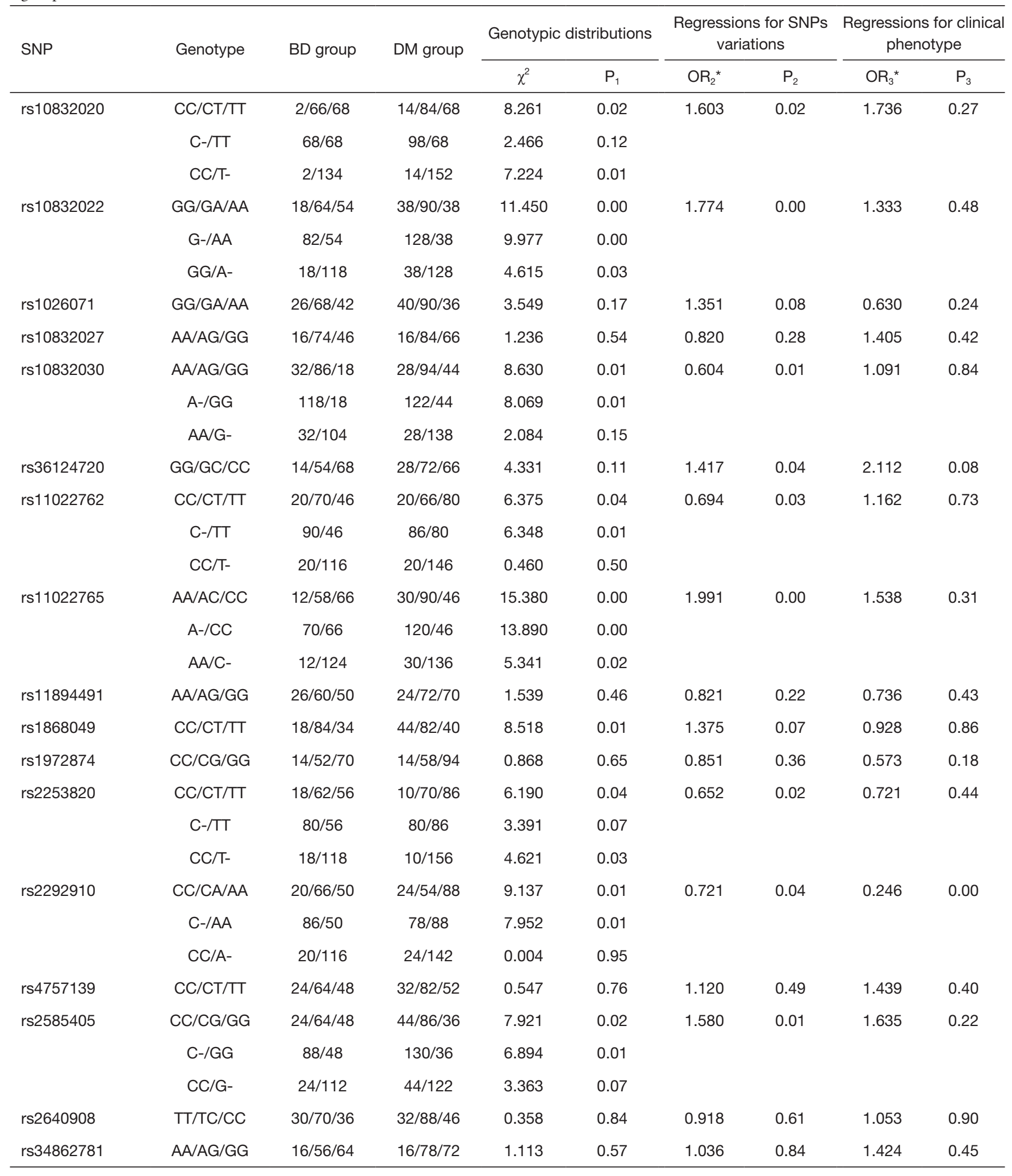

Table 3 (continued) 
Table 3 (continued)

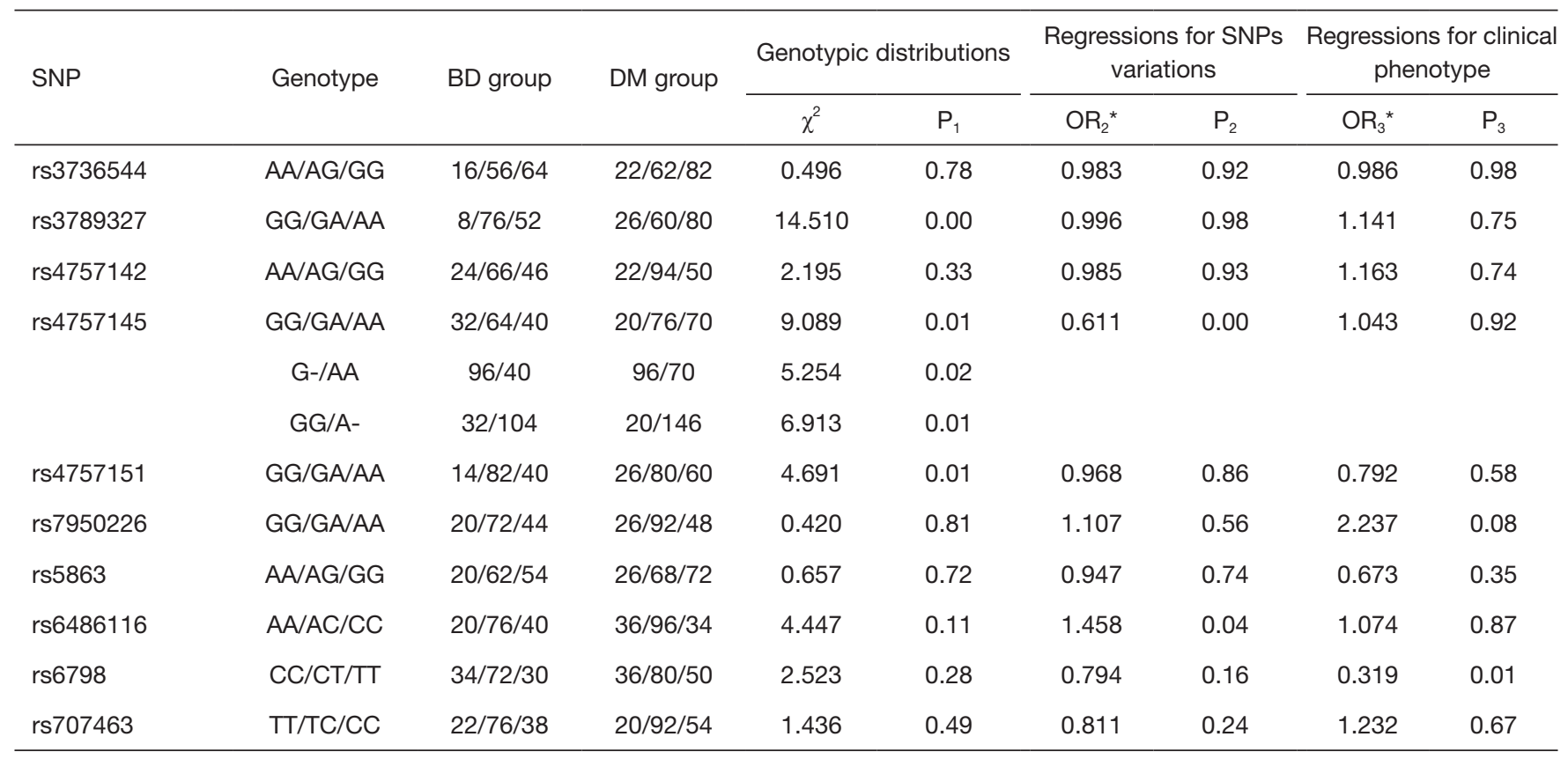

* OR (odds ratio): risk value of DM group compared with BD group.

The genotypic distribution of rs2292910 was statistically different between the DM group and BD group $\left(\chi^{2}=9.137\right.$, $\mathrm{P}=0.01)$. Moreover, after both the regression including each SNP genotypic distributions, and clinical phenotypic regression including gender and age, the difference was still statistically significant $(\mathrm{OR}=0.712, \mathrm{P}=0.04$ for the former, $\mathrm{OR}=0.246, \mathrm{P}=0.00$ for the latter). The frequency of the AA genotype was significantly higher in the DM group (53.0\%) than that in the BD group $(36.8 \%)\left(\chi^{2}=7.952, \mathrm{P}=0.01\right)$.

The genotype distribution of rs 11022762 was also statistically different between the DM group and BD group $\left(\chi^{2}=6.375, \mathrm{P}=0.04\right)$, and after regression including each SNP genotypic distributions, the difference was still statistically significant $(\mathrm{OR}=0.694, \mathrm{P}=0.03)$. However, after the clinical phenotypic regression including gender and age, the difference was not statistically significant. The frequency of the TT genotype in the DM group (48.2\%) was significantly higher than that in the BD group (33.8\%) $\left(\chi^{2}=6.348, \mathrm{P}=0.01\right)$.

The genotypic distribution of rs10832020 was statistically different between the DM group and BD group $\left(\chi^{2}=8.261, \mathrm{P}=0.02\right)$, and after regression including each SNP genotypic distributions, the difference was still statistically significant $(\mathrm{OR}=1.03, \mathrm{P}=0.02$ ). The frequency of the $\mathrm{CC}$ genotype in the DM group was significantly higher than in the BD group (8.4\% for the former and $1.5 \%$ for the latter; $\left.\chi^{2}=7.224, \mathrm{P}=0.01\right)$.

Rs10832022, rs11022765, and rs1 1022762 had LD through LD analysis (Figure 1C), and haplotype analysis showed that the distribution frequency of rs10832022-A, rs11022765-C, and rs11022762-C haplotype in the DM group $(31.2 \%)$ was significantly lower than in the $\mathrm{BD}$ group $(40.4 \%)\left(\chi^{2}=5.309, \mathrm{P}=0.02, \mathrm{OR}=0.674,95 \% \mathrm{CI}\right.$ : $0.482-0.944)$. The distribution frequency of rs10832022-G, rs11022765-A, and rs11022762-T haplotype in the DM group $(44.4 \%)$ was significantly higher than in the BD group $(30.1 \%) \quad\left(\chi^{2}=13.489, \mathrm{P}=0.0002, \mathrm{OR}=1.878,95 \% \mathrm{CI}\right.$ : $1.339-2.633)$.

In addition, rs10832020 and rs6486116 also had LD through LD analysis (Figure 1C), and haplotype analysis showed that the distribution frequency of rs10832020-C and rs6486116-A haplotype in the DM group (33.7\%) was significantly higher than in the $\mathrm{BD}$ group $(25.7 \%)\left(\chi^{2}=4.545\right.$, $\mathrm{P}=0.03, \mathrm{OR}=1.469,95 \%$ CI: $1.031-2.094)$.

\section{Discussion}

Both the frequencies of the rs $10832022 \mathrm{G}$ allele/Ggenotype and rs11022765 A allele/A-genotype were significantly higher in patients with DM than in those 
with BD. This result suggests that the two alleles might be closely related to the occurrence of type $2 \mathrm{DM}$. The frequency of the rs11022762 TT genotype, as well as that of the rs10832022-G, rs11022765-A, and rs11022762-T haplotype was both significantly higher in patients with DM than in those with BD. Rs10832022, rs11022765, and rs11022762 were all located in the ARNTL gene intron 2 region. The ARNTL gene, also known as the BMAL1 gene, is one of the core components of the biological rhythm oscillator of the body, and the transcription factor protein encoded by ARNTL can generate circadian rhythm through its positive and negative feedback pathways (21). The ARNTL gene was also thought to be associated with blood pressure fluctuations and metabolic syndrome. Previous animal experiments have found that some genetic variants of this gene might induce spontaneous hypertension and significantly affect the transcriptional activation of Gata4 in rats (22), while mice with ARNTL gene knockout developed endothelial dysfunction, leading to increased vascular damage (23). In addition, mice with ARNTL gene knockout also had abnormal glucose tolerance and significantly decreased insulin secretion in vivo, and some mutations in the ARNTL gene could increase the risk of hypertension and type $2 \mathrm{DM}$ in individuals (22). Moreover, diabetic mice showed a disturbance of heart rate, blood pressure, and circadian rhythm when expression of the ARNTL gene in arteries was inhibited (24). Our results add support to the observation that the ARNTL gene, especially the variations in its intron region, may be related to the occurrence and development of type $2 \mathrm{DM}$.

It is worth noting that the genotypic distributions of rs10832022 and rs11022765 in ANRTL gene also showed significant differences between patients with BD and controls. Frequencies of the rs10832022 GG genotype and rs11022765 AA genotype were much lower in the BD group, and the frequency of rs10832022-G, rs11022765-A, and rs11022762-T haplotype was also significantly lower in the BD group. Some SNP variations in the ARNTL gene have been linked to insomnia in women (25). A recent study further found that sleep could drive the rhythmic migration of the ARNTL gene in splenic monocytes and improve the function of immune cells (26), suggesting a role in the regulation of sleep rhythm of this gene. As sleep disorders are common in patients with $\mathrm{DM}$ or $\mathrm{BD}$, our results demonstrated to some extent the hypothesis that DM and $\mathrm{BD}$ are rhythm disorders. Variations in the intron region of the ARNTL gene may be one of the genetic backgrounds of emotional rhythm disorder or glucometabolic rhythm disorder in individuals. Although the intron region of the gene was not involved in the transcription and translation of the corresponding RNA, it might still affect the linear expression of the gene and the selective splicing of the transcription and translation, affecting the occurrence of rhythm disorders (27). In the future, with the combination of downstream functional changes, we will further explore the role of the ARNTL gene in the occurrence and development of rhythm abnormalities.

Cryptochrome 2 gene (CRY2), a transcriptional inhibitor, is one component in the negative regulation loop of biological rhythm, playing an important role in maintaining circadian stability and regulating cell cycle and DNA repair. It is widely expressed in the liver, pancreases, heart, and other tissues (28). The expression of this gene was downregulated in some tumor tissues (29) and might influence the occurrence of breast cancer through hormone-mediated signal transduction (30). A previous study has found that rs2292910 SNP of this gene may affect bone formation and resorption, and its $\mathrm{C}$ allele might be a protective factor in the pathogenesis of osteoporosis (31). We found that the frequency of the rs2292910 CC genotype was significantly higher in patients with $\mathrm{BD}$ than in controls, and frequency of the AA genotype was significantly higher in patients with type $2 \mathrm{DM}$ than with BD. Furthermore, the above differences were not affected by the clinical phenotype of diseases and other detected SNPs variations. Therefore, rs2292910 in CRY2 gene might be used as a genetic marker to predict the risk of somatic or emotional rhythm disorder in individuals. The CC genotype may be the risk genotype for $\mathrm{BD}$ (an emotional rhythm disorder), while the AA genotype may be a risk predictor for individuals to differentiate into type $2 \mathrm{DM}$ (a glucose metabolism rhythm disorder) rather than the emotional rhythm disorder. As rs2292910 was located in the exon 13 region of the CRY2 gene 3'-UTR, its variation might regulate the expression of CRY2, thereby affecting the individual's rhythmic function. The rs11605924 SNP of the CRY2 gene was thought to be associated with increased fasting glucose level in the general population and was a risk factor for metabolic syndrome. It was further speculated that the CRY2 gene might be related to glucose metabolism and energy balance and affect the susceptibility of individuals to obesity (32). Our results further support the view that the CRY2 gene may play an important role in the development of type 2 DM, as its different genetic variants had a certain predictive value for the risk of somatic glucose metabolism rhythm disturbance.

This study showed that the rs7950226 G allele carriers 
were much more prevalent in patients with type $2 \mathrm{DM}$ than in controls, and the OR value reached 2.909, suggesting the G- genotype was a risk genotype for type 2 DM. Rs7950226 is also located in the intron 1 region of the ARNTL gene, and its A allele was found to associated with the occurrence of type $2 \mathrm{DM}$ in a Greek population (33), contrary to our result. While this contradiction may be attributed to ethnic differences, both results suggest the genetic variability in the intron region of the ARNTL gene may be the rhythmrelated genetic background for the occurrence of type 2 DM. In addition, rs10832020 and rs6486116, two SNPs also located in the intron 1 region of the ARNTL gene and having significantly different distributions between patients with type2 DM and with BD, had no LD with rs7950226. Therefore, we believe that the different genotypic variation of rs7950226 may be an independent risk factor associated with rhythm heredity in the occurrence of type $2 \mathrm{DM}$.

Rs1972874 and rs36124720 were both located in the intron region of PER2 gene and had a strong LD in this study. The distribution frequency of the G-G haplotype in patients with type $2 \mathrm{DM}$ was significantly higher than in controls, with an OR value of 1.577 , suggesting a high-risk genotype for the occurrence of type 2 DM. The intron region of PER2 gene may be correlated with the susceptibility to type 2 DM. PER2 belongs to the mammalian Period family, which can regulate its own transcription in both direct and indirect ways, and form an endogenous biological clock with CRY/BMAL1 and CLOCK to regulate body rhythm (28). Sevoflurane could cause PER2 to lose its rhythm, which could be restored by light (34). The serum cortisol peak was delayed in the diabetic depression model rats, and the expression of PER2 in SCN was up-regulated compared with a control model, which suggested that overexpression of the PER2 gene might inhibit the positive activation process and change the HPA axis rhythm of the body, leading to fluctuations of blood sugar and mood (35). Although the intron region did not participate in the final expression of PER2, its different gene mutations might still influence the abnormal regulation of downstream rhythm through the intervention of splicing (36). Further studies will focus on the effect of PER2 gene variations to the dysrhythmia of body glucose metabolism by combining epigenetics.

Unfortunately, this study did not find any risk allele for type $2 \mathrm{DM}$ or $\mathrm{BD}$. On this basis, we consider that only a single SNP mutation might not be enough to cause these diseases. As abnormal expressions of some rhythm genes are found in many chronic non-infectious diseases, including type $2 \mathrm{DM}(37)$, it is necessary to combine epigenetic indicators and other clinical rhythm changes to further explore the genetic mechanism of different rhythm disorders. It is worth noting that the genotypic distributions of rs10832022, rs11022765, and rs2292910 SNPs were not only significantly different between patients with type 2 $\mathrm{DM}$ and those with $\mathrm{BD}$, but also between patients with $\mathrm{BD}$ and controls. This suggests that the three SNPs may be relatively independent genetic mutation sites for $\mathrm{BD}$, being worthy of further study.

In conclusion, the present study preliminarily demonstrates that some variations of rhythm genes, especially the SNPs located in rhythm gene ARNTL intron region, CRY2 3'-UTR and PER2 intron region are associated with the susceptibility to type $2 \mathrm{DM}$ or BD. Meanwhile, these variations may predict the risk of developing glucometabolic or emotional dysrhythmia in individuals, and be the specific rhythm genetic background of the two diseases. These results preliminarily verified the theoretical hypothesis of "stress-dysrhythmia" about the mechanisms of chronic non-infectious diseases (17). Based on the different rhythm genetic variations, individuals may have different biological rhythm dysfunction and lead to different diseases with various stress factors.

Our study not only suggests both type $2 \mathrm{DM}$ and BD could be considered as rhythmic disorders with different genetic backgrounds of rhythm, but also is helpful for the early prediction of individual susceptibility to physical or emotional rhythm disorder and for early intervention.

\section{Acknowledgments}

We thank the patients and their family members for participating in this study. We also thank Dr. Hao Xu, Dr. Fang Luo, Dr. Xi Zhang, and Dr. Min Zhu for their cooperation in collecting samples.

Funding: This study was supported by The Youth Foundation of Sichuan Provincial People's Hospital (Number: 2017QN13), and the Chinese Academy of Medical Sciences central public research base platform construction project (Number: 2018PT31049) supported the cost of DNA extraction and genotyping. Funds also paid the printing fees for clinical protocols.

\section{Footnote}

Reporting Checklist: The authors have completed the STREGA reporting checklist. Available at https://dx.doi. 
org/10.21037/atm-21-4803

Data Sharing Statement: Available at https://dx.doi. org/10.21037/atm-21-4803

Conflicts of Interest: All authors have completed the ICMJE uniform disclosure form (available at https://dx.doi. org/10.21037/atm-21-4803). The authors have no conflicts of interest to declare.

Ethical Statement: The authors are accountable for all aspects of the work in ensuring that questions related to the accuracy or integrity of any part of the work are appropriately investigated and resolved. All procedures performed in this study involving human participants were in accordance with the Declaration of Helsinki (as revised in 2013). The study was approved by Institutional Ethics Committee of the Sichuan Provincial People's Hospital [Ethic review (Research) No. 18.2018]. All methods were performed in accordance with the relevant guidelines and regulations, and written informed consent was obtained from each participant.

Open Access Statement: This is an Open Access article distributed in accordance with the Creative Commons Attribution-NonCommercial-NoDerivs 4.0 International License (CC BY-NC-ND 4.0), which permits the noncommercial replication and distribution of the article with the strict proviso that no changes or edits are made and the original work is properly cited (including links to both the formal publication through the relevant DOI and the license). See: https://creativecommons.org/licenses/by-nc-nd/4.0/.

\section{References}

1. Loudon AS. Circadian biology: a 2.5 billion year old clock. Curr Biol 2012;22:R570-1.

2. Albrecht U. Timing to perfection: the biology of central and peripheral circadian clocks. Neuron 2012;74:246-60.

3. Ciarleglio CM, Ryckman KK, Servick SV, et al. Genetic differences in human circadian clock genes among worldwide populations. J Biol Rhythms 2008;23:330-40.

4. Schloss MJ, Horckmans M, Nitz K, et al. The time-ofday of myocardial infarction onset affects healing through oscillations in cardiac neutrophil recruitment. EMBO Mol Med 2016;8:937-48.

5. Golden SH. A review of the evidence for a neuroendocrine link between stress, depression and diabetes mellitus. Curr
Diabetes Rev 2007;3:252-9.

6. Huang SM, Quan QF. Study on the interaction and pathogenesis of type 2 diabetes and anxiety and depressive disorder. World Latest Medicine Information 2019;19:134-5.

7. Tokat B, Kanca-Demirci D, Gul N, et al. Determination of genetic changes of Rev-erb beta and Rev-erb alpha genes in Type 2 diabetes mellitus by next-generation sequencing. Gene 2020;763:145058.

8. Corella D, Asensio EM, Coltell O, et al. CLOCK gene variation is associated with incidence of type-2 diabetes and cardiovascular diseases in type-2 diabetic subjects: dietary modulation in the PREDIMED randomized trial. Cardiovasc Diabetol 2016;15:4.

9. Huang Y, Wang Y, Wang H, et al. Prevalence of mental disorders in China: a cross-sectional epidemiological study. Lancet Psychiatry 2019;6:211-24.

10. Liu M, Meng Y, Wei W, et al. Relationship between circadian rhythm related brain dysfunction and bipolar disorder. Nan Fang Yi Ke Da Xue Xue Bao 2020;40:822-7.

11. Moreira J, Geoffroy PA. Lithium and bipolar disorder: Impacts from molecular to behavioural circadian rhythms. Chronobiol Int 2016;33:351-73.

12. Yegin Z, Sarisoy G, Erguner Aral A, et al. For whom the circadian clock ticks? Investigation of PERIOD and CLOCK gene variants in bipolar disorder. Chronobiol Int 2021;38:1109-19.

13. Havermans R, Nicolson NA, Berkhof J, et al. Patterns of salivary cortisol secretion and responses to daily events in patients with remitted bipolar disorder. Psychoneuroendocrinology 2011;36:258-65.

14. Seleem MA, Merranko JA, Goldstein TR, et al. The longitudinal course of sleep timing and circadian preferences in adults with bipolar disorder. Bipolar Disord 2015;17:392-402.

15. Turek FW, Joshu C, Kohsaka A, et al. Obesity and metabolic syndrome in circadian Clock mutant mice. Science 2005;308:1043-5.

16. Charrier A, Olliac B, Roubertoux P, et al. Clock Genes and Altered Sleep-Wake Rhythms: Their Role in the Development of Psychiatric Disorders. Int J Mol Sci 2017;18:938.

17. Sun XL, Zhang X. Bipolar disorder and its atypical symptoms: A consensus of recognition and optimal treatment (the second edition). Beijing: Science Press, 2017.

18. Li Z, Yang XR, Li T. Research progress of comorbidities between bipolar disorder and somatic diseases. Chinese Journal of Nervous and Mental Diseases 2015;41:122-5. 
19. First MB, Spitzer RL, Gibbon M, et al. Structured clinical interview for DSM-IV-TR axis I disorders, research version, non-patient edition (SCID-I/NP). New York: Biometrics Research, New York State Psychiatric Institute, 2002.

20. Shi YY, He L. SHEsis, a powerful software platform for analyses of linkage disequilibrium, haplotype construction, and genetic association at polymorphism loci. Cell Res 2005;15:97-8.

21. Takahashi JS. Molecular components of the circadian clock in mammals. Diabetes Obes Metab 2015;17 Suppl 1:6-11.

22. Woon PY, Kaisaki PJ, Bragança J, et al. Aryl hydrocarbon receptor nuclear translocator-like (BMAL1) is associated with susceptibility to hypertension and type 2 diabetes. Proc Natl Acad Sci U S A 2007;104:14412-7.

23. Anea CB, Zhang M, Stepp DW, et al. Vascular disease in mice with a dysfunctional circadian clock. Circulation 2009;119:1510-7.

24. Huang Q, Xia ZY, Lei SQ. Effect of diabetes mellitus on diurnal expression of BMAL1/PER2 myocardial clock gene. Medical Recapitulate 2018;24:4953-4957+4963.

25. Ziv-Gal A, Flaws JA, Mahoney MM, et al. Genetic polymorphisms in the aryl hydrocarbon receptor-signaling pathway and sleep disturbances in middle-aged women. Sleep Med 2013;14:883-7.

26. Hahn J, Günter M, Schuhmacher J, et al. Sleep enhances numbers and function of monocytes and improves bacterial infection outcome in mice. Brain Behav Immun 2020;87:329-38.

27. Angelousi A, Kassi E, Nasiri-Ansari N, et al. Clock genes alterations and endocrine disorders. Eur J Clin Invest 2018;48:e12927.

28. Gallego M, Virshup DM. Post-translational modifications regulate the ticking of the circadian clock. Nat Rev Mol Cell Biol 2007;8:139-48.

Cite this article as: Min W, Tang N, Zou Z, Chen Y, Zhang X, Huang Y, Wang J, Zhang Y, Zhou B, Sun X. A panel of rhythm gene polymorphisms is involved in susceptibility to type 2 diabetes mellitus and bipolar disorder. Ann Transl Med 2021;9(20):1555. doi: 10.21037/atm-21-4803
29. Mannic T, Meyer P, Triponez F, et al. Circadian clock characteristics are altered in human thyroid malignant nodules. J Clin Endocrinol Metab 2013;98:4446-56.

30. Guardavaccaro D, Clevers H. Wnt/ $\beta$-catenin and MAPK signaling: allies and enemies in different battlefields. Sci Signal 2012;5:pe15.

31. Li Y, Zhou J, Wu Y, et al. Association of osteoporosis with genetic variants of circadian genes in Chinese geriatrics. Osteoporos Int 2016;27:1485-92.

32. Dupuis J, Langenberg C, Prokopenko I, et al. New genetic loci implicated in fasting glucose homeostasis and their impact on type 2 diabetes risk. Nat Genet 2010;42:105-16.

33. Pappa KI, Gazouli M, Anastasiou E, et al. The major circadian pacemaker ARNT-like protein-1 (BMAL1) is associated with susceptibility to gestational diabetes mellitus. Diabetes Res Clin Pract 2013;99:151-7.

34. Li JT. Effects of sevoflurane on the expression of PER2 gene in mice and the regulation of light. Shanghai: People's Liberation Army Naval Medical University, 2019.

35. Hong XJ, Huang BL, Hong JX. Effects of acupuncture on serum CORT circadian rhythm and PER1 and PER2 gene expression in SCN of diabetic depression model rats. Liaoning Journal of Traditional Chinese Medicine 2018;45:483-6.

36. Shumay E, Fowler JS, Wang GJ, et al. Repeat variation in the human PER2 gene as a new genetic marker associated with cocaine addiction and brain dopamine D2 receptor availability. Transl Psychiatry 2012;2:e86.

37. McCarthy MJ, Wei H, Marnoy Z, et al. Genetic and clinical factors predict lithium's effects on PER2 gene expression rhythms in cells from bipolar disorder patients. Transl Psychiatry 2013;3:e318.

(English Language Editor: B. Draper) 


\section{Supplementary}

Table S1 Basic information of the 29 selected SNP sites

\begin{tabular}{|c|c|c|c|c|c|}
\hline SNP & $\mathrm{CHR}^{*}$ & Gene Name & Position & $\mathrm{MAF}^{\#}$ & ene pool) \\
\hline rs4757139 & 11 & ARNTL & 13300456 & $\mathrm{C}$ & 0.423 \\
\hline rs4757142 & 11 & ARNTL & 13325695 & $A$ & 0.416 \\
\hline rs4757145 & 11 & ARNTL & 13331324 & $\mathrm{G}$ & 0.435 \\
\hline rs1026071 & 11 & ARNTL & 13364712 & $\mathrm{G}$ & 0.488 \\
\hline rs10832020 & 11 & ARNTL & 13321343 & C & 0.313 \\
\hline rs10832022 & 11 & ARNTL & 13339272 & $\mathrm{G}$ & 0.445 \\
\hline rs10832027 & 11 & ARNTL & 13357183 & $A$ & 0.399 \\
\hline rs10832030 & 11 & ARNTL & 13399791 & $\mathrm{G}$ & 0.490 \\
\hline rs11022762 & 11 & ARNTL & 13335926 & C & 0.392 \\
\hline rs11022765 & 11 & ARNTL & 13345115 & $A$ & 0.373 \\
\hline rs11894491 & 2 & PER2 & 239198325 & $A$ & 0.341 \\
\hline rs1868049 & 11 & ARNTL & 13383682 & C & 0.454 \\
\hline rs1972874 & 2 & PER2 & 239178402 & C & 0.315 \\
\hline rs2253820 & 17 & PER1 & 8048169 & C & 0.310 \\
\hline rs2292910 & 11 & CRY2 & 45903613 & C & 0.325 \\
\hline rs2585405 & 17 & PER1 & 8046772 & C & 0.440 \\
\hline rs2640908 & 1 & PER3 & 7889941 & $\mathrm{~T}$ & 0.486 \\
\hline rs34862781 & 11 & ARNTL & 13305263 & $A$ & 0.339 \\
\hline rs36124720 & 2 & PER2 & 239155971 & G & 0.361 \\
\hline rs3736544 & 4 & CLOCK & 56309992 & $A$ & 0.334 \\
\hline rs3789327 & 11 & ARNTL & 13385316 & $\mathrm{G}$ & 0.332 \\
\hline rs4757151 & 11 & ARNTL & 13392213 & $\mathrm{G}$ & 0.399 \\
\hline rs55794336 & 12 & CRY1 & 107445977 & $\mathrm{G}$ & 0.423 \\
\hline rs5863 & 4 & CLOCK & 56296907 & $A$ & 0.404 \\
\hline rs6486116 & 11 & ARNTL & 13319838 & $A$ & 0.490 \\
\hline rs6798 & 11 & CRY2 & 45904477 & C & 0.452 \\
\hline rs707463 & 1 & PER3 & 7850062 & $\mathrm{~T}$ & 0.450 \\
\hline rs7950226 & 11 & ARNTL & 13318139 & $\mathrm{G}$ & 0.392 \\
\hline
\end{tabular}

CHR, chromosome; MAF, minor allele frequency. 
Table S2 Primer sequences of SNPs detected

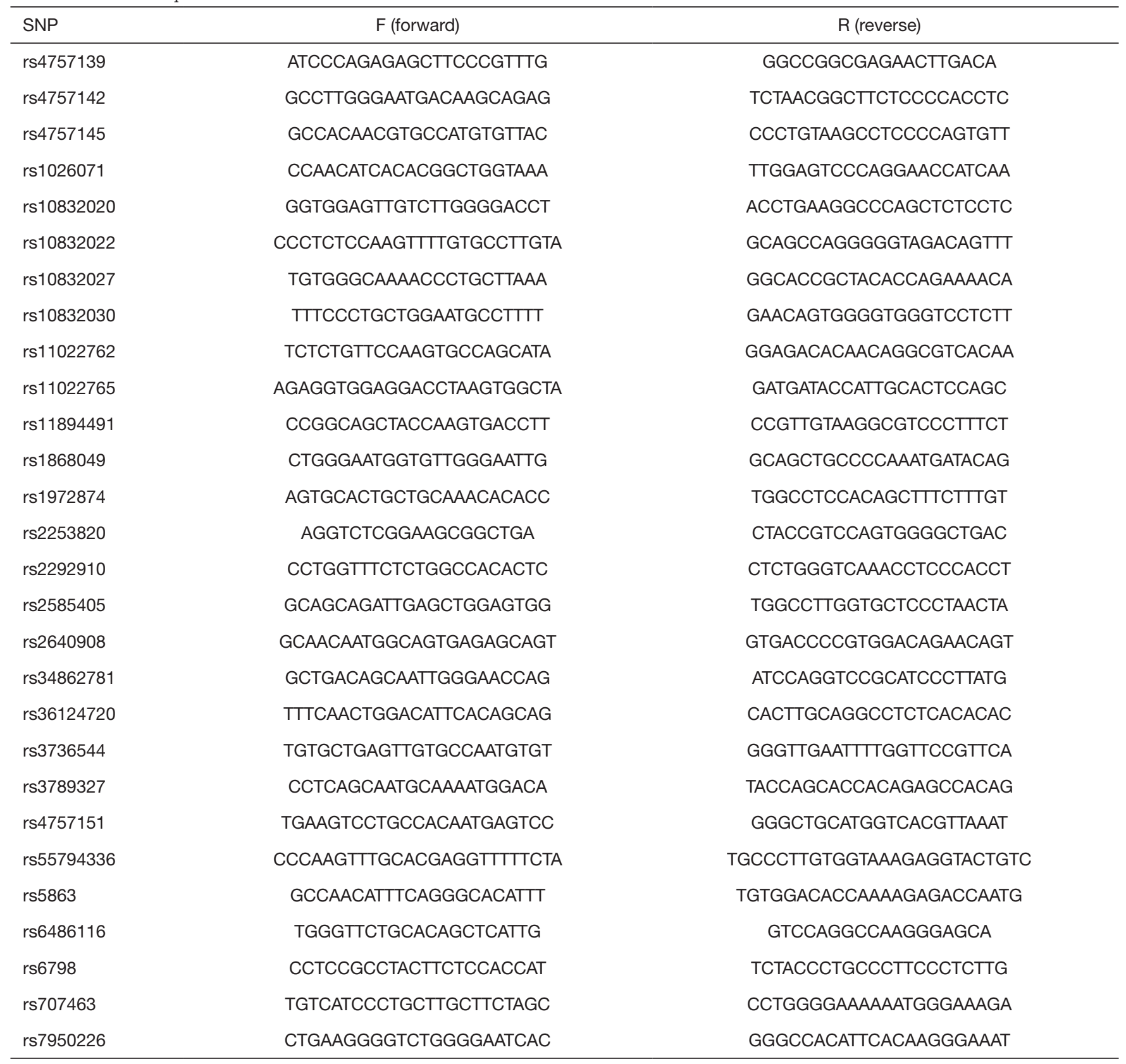


Table S3 Hardy-Weinberg equilibrium (HWE) analysis

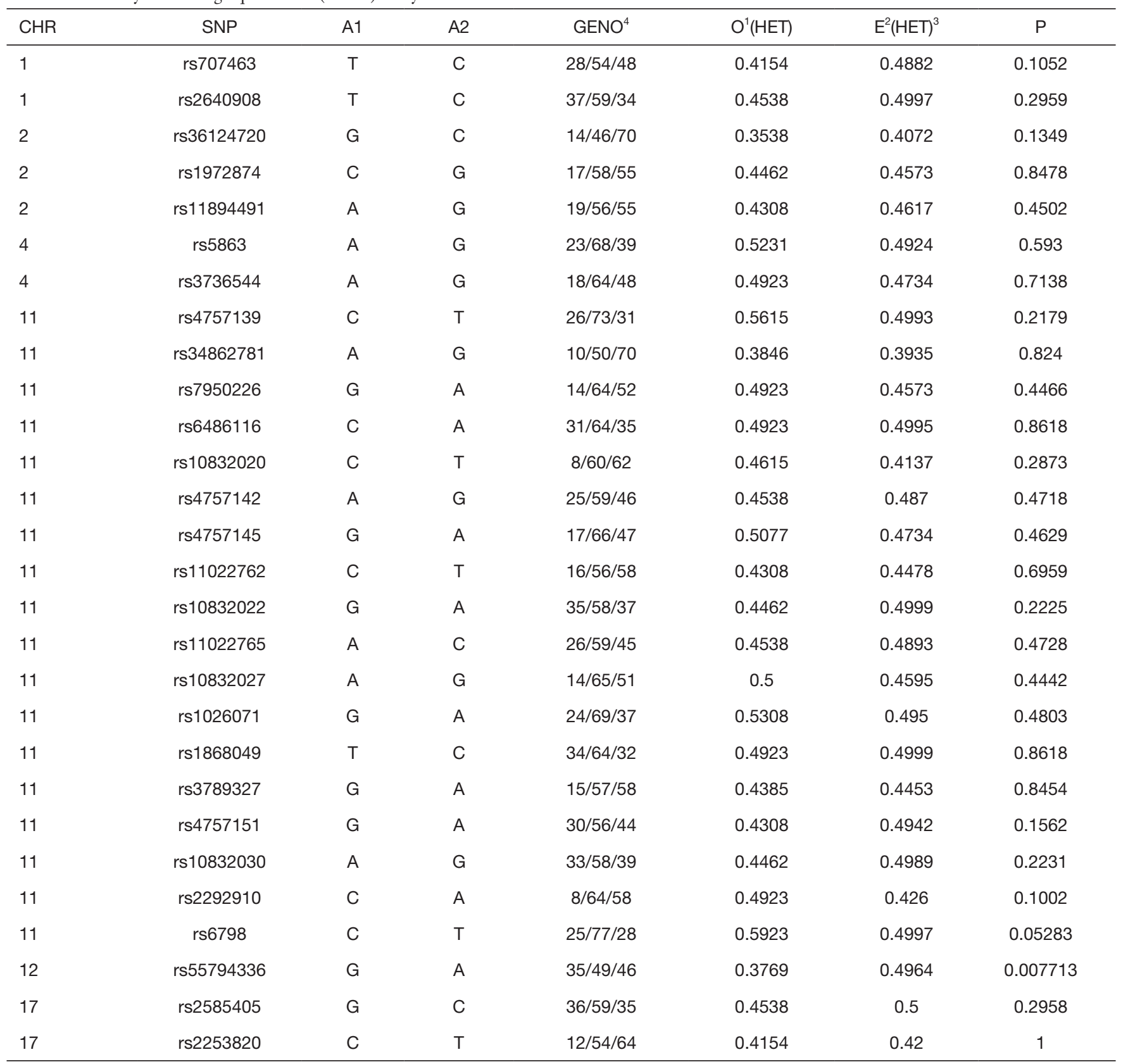

${ }^{1} \mathrm{O}$ : observed frequency; ${ }^{2} \mathrm{E}$ : expected frequency; ${ }^{3} \mathrm{HET}$ : heterozygosity; ${ }^{4} \mathrm{GENO}$ : genotype. 